\title{
Transcatheter Mitral Valve-in-Valve Procedure for Dilated Cardiomyopathy Concomitant with Prosthetic Mitral Valve Dysfunction in a Heart Transplant Candidate
}

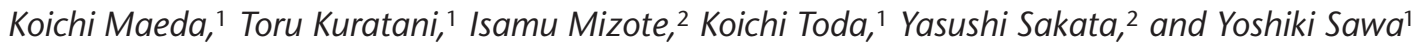 \\ ${ }^{1}$ Department of Cardiovascular Surgery, Osaka University Graduate School of Medicine, Suita, Osaka, Japan \\ ${ }^{2}$ Department of Cardiology, Osaka University Graduate School of Medicine, Suita, Osaka, Japan
}

Received: December 11, 2019; Accepted: March 16, 2020

Objective: The surgical treatment of severe mitral regurgitation concomitant with dilated cardiomyopathy (DCM) is challenging because of poor heart function. Herein, we have reported a successful transcatheter mitral valve-in-valve (TM-ViV) implantation (valve-in-valve procedure) in a heart transplant candidate.

Case Presentation: A 66-year-old man who suffered from DCM underwent mitral valve replacement for mitral regurgitation 7 years ago and was registered as a candidate for heart transplantation (status 2) at 65 years of age. He suffered from dyspnea on effort (New York heart association functional classification (NYHA) III) and his echocardiography revealed prosthetic mitral valve dysfunction (severe regurgitation). Because the surgical risk for prosthetic valve dysfunction was high (society of thoracic surgeons (STS) score: 11.2\%), he was enrolled for on-pump TM-ViV procedure. The intraoperative and postoperative courses were uneventful, and echocardiography at the 18-month follow-up revealed low mean pressure gradient $(4.2 \mathrm{mmHg}$ ) transmitral bioprosthesis and no paravalvular leakage.

Conclusions: On-pump transcatheter mitral valve implantation may be an important treatment option for severely injured left ventricular (LV) function with a failed bioprosthesis.

Keywords: mitral regurgitation, prosthesis, heart transplantation

\section{Introduction}

The surgical treatment for severe mitral regurgitation concomitant with dilated cardiomyopathy (DCM) is challenging because of the poor heart function. Here, we have reported a successful case of treatment with transcatheter mitral valve-in-valve (TM-ViV) implantation (valve-invalve procedure) for prosthetic mitral valve dysfunction (severe mitral regurgitation) concomitant with DCM.

Corresponding author: Yoshiki Sawa. Department of Cardiovascular Surgery, Osaka University Graduate School of Medicine, 2-2 Yamadaoka, Suita, Osaka 565-0871, Japan

Email: sawa-p@surg1.med.osaka-u.ac.jp

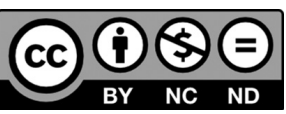

This work is licensed under a Creative Commons Attribution-NonCommercialNoDerivatives International License.

C)2020 Journal of Transcatheter Valve Therapies Society

\section{Case Report}

A 66-year-old man who suffered from DCM underwent mitral valve replacement with a $27-\mathrm{mm}$-perimount CEP (Edwards Lifesciences, Irvine, CA, USA) for mitral regurgitation and myoblast cell sheet implantation 7 years ago. ${ }^{1)}$ Furthermore, he was registered as a candidate for heart transplantation (status 2) at 65 years of age. However, he suffered from dyspnea on effort (New York heart association functional classification (NYHA) III), and his echocardiography revealed dilated left ventricular (LV end-diastolic diameter [LVDd]/LV end-systolic diameter [LVDs]: 71/67 $\mathrm{mm}$ ), poor LV function (23\%), and prosthetic mitral valve dysfunction (severe regurgitation) (Fig. 1A). Catheterization revealed high pulmonary capillary wedge pressure (PCWP) $(25 \mathrm{mmHg})$, high pulmonary artery pressure (51/28 [38] $\mathrm{mmHg}$ ], and low output (CI: $\left.2.17 \mathrm{~L} / \mathrm{min} / \mathrm{m}^{2}\right)$. 

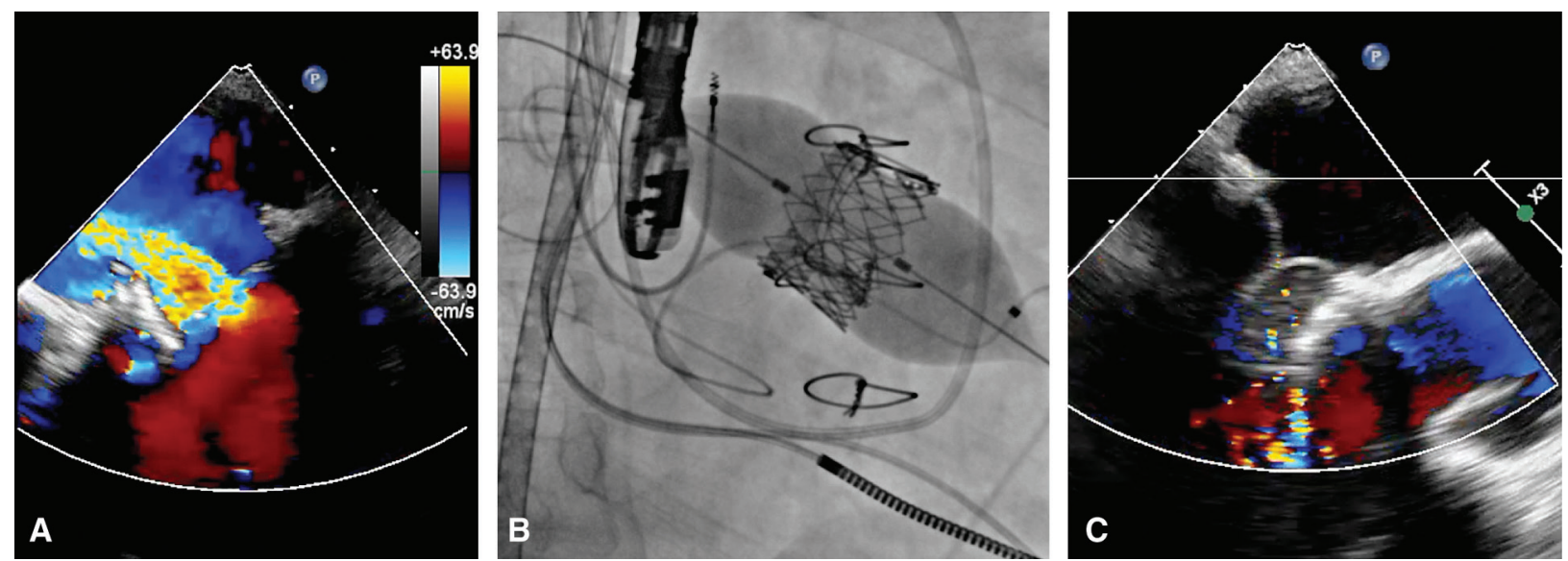

Fig. 1 Preoperative and postoperative mitral prosthetic valve regurgitation. Preoperative echocardiography revealed severe mitral prosthetic valve regurgitation (A). The $29 \mathrm{~mm}$-SAPIEN XT was implanted on the accurate position (B). No regurgitation after transcatheter mitral valve implantation $(\mathbf{C})$.
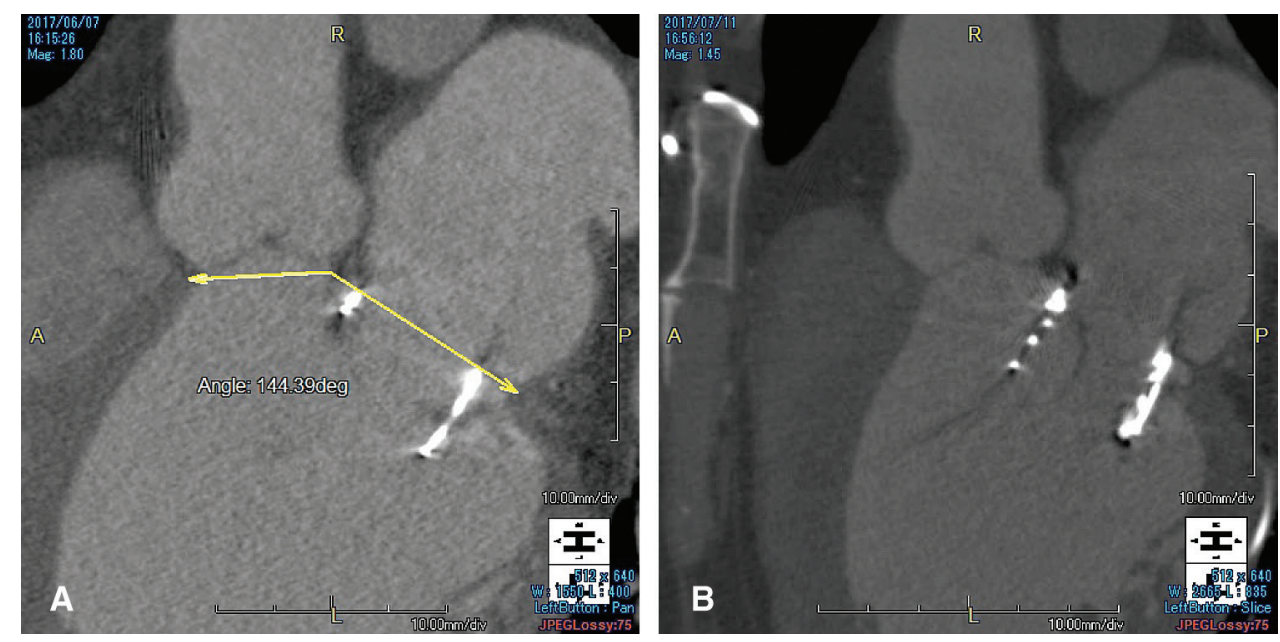

Fig. 2 Preoperative aortomitral angulation on cardiac CT. In our patient, the risk for neo-LVOT stenosis was expected to be low because aortomitral angulation was sufficient $\left(144^{\circ}\right)$ (A: preoperative CT, B: postoperative CT). CT: computed tomography; LVOT: left ventricular outflow tract

Because the surgical risk for prosthetic valve dysfunction was high (society of thoracic surgeons (STS) score: 11.2\%), the patient was enrolled for $\mathrm{TM}-\mathrm{ViV}$ procedure. Because of severely injured LV function, a cardiopulmonary support was considered to be useful to reduce the end-diastolic pressure after rapid pacing and to stabilize the hemodynamic status. The study protocol (valve-in-valve procedure for prosthetic valve dysfunction) was approved by the Institutional Review Board of Osaka University, and the patient provided a written informed consent.

Procedure was performed under general anesthesia in a hybrid operating room by a multidisciplinary heart team. We established cardiopulmonary bypass (CPB) with the outflow right-subclavian artery and inflow right-femoral vein to reduce the end-diastolic pressure before and after a rapid pacing. In addition, we performed the trans-apical transcatheter procedure via the sixth intercostal space in the standard manner. Before the apical puncture, we palpated with the finger and looked for the best trajectory on transthoracic echocardiogram to reach the mitral prosthetic valve. After the apical puncture, we placed the Safari wire (S size) (Boston Scientific, Marlborough, MA, USA) in the left atrium. The 29-mm-reversed-crimped SAPIEN XT (Edwards Lifesciences, Irvine, CA, USA) was delivered using Ascendra delivery system (Edwards Lifesciences, Irvine, CA, USA). Prior balloon valvuloplasty was not performed. The SAPIEN valve was implanted at the accurate position under rapid ventricular 
pacing (Fig. 1B). The CPB was smoothly removed with a small dose of inotropes. Transesophageal echocardiography revealed no paravalvular leakage (Fig. 1C). The patient was extubated at $4 \mathrm{~h}$ after the operation. The postoperative course was uneventful. The weaning of inotropes and the administration of beta-blocker were smoothly performed. The patient was discharged at 14 days after operation without any complication. At the 18-month follow-up, no cardiac events were noted and the echocardiography revealed no paravalvular leakage and a low mean pressure gradient $(4.2 \mathrm{mmHg})$ transmitral bioprosthesis.

\section{Discussion}

Surgery for severe functional mitral regurgitation (FMR) provides better long-term outcomes in patients with DCM because persistent moderate/severe FMR with DCM is associated with poor clinical outcomes, despite sufficient medical therapy. ${ }^{2)}$ However, surgery, especially redocardiovascular surgery, in patients with FMR and DCM has generally a high risk, and a recurrence rate of mitral valve repair or mitral valve replacement of $5-10 \%$ at 10 years after surgery has also been reported. On the other hand, left ventricular assist devices (LVADs) improve the survival and quality of life for most patients with advanced heart failure. However, prolonged support with LVADs due to the shortage of donors can lead to VAD-specific/ related complications, including infections and stroke. ${ }^{3)}$ Therefore, other treatment options have been considered mandatory in case of donor shortage. $\mathrm{TM}-\mathrm{ViV}$ procedure has emerged as an alternative for redo-mitral intervention after mitral valve repair or mitral valve replacement. ${ }^{4)}$

However, it has some disadvantages, for instance, the development of left ventricular obstruction.. ${ }^{5)}$ Blanke et al. $\left.{ }^{6}\right)$ mentioned that TM-ViV creates "neo-left ventricular outflow tract (LVOT)" anteriorly through basal septum and posteriorly through the deflected bioprosthetic leaflets and transcatheter heart valve struts. Narrow neoLVOT develops by various anatomical and device-related factors. LVOT obstruction results in patients with sharper aortomitral angulation, smaller LV, longer anterior leaflet, and/or larger septal bulge. In the present case, the risk for neo-LVOT stenosis was expected to be low because of large LV dimension (LVDd/LVDs: 71/67 mm) and an obtuse aortomitral angulation (144\%) (Fig. 2).

Furthermore, the intraoperative and postoperative courses were uneventful despite severely injured LV function. This was because an intraoperative cardiopulmonary support was established to reduce the end-diastolic pressure, especially after rapid ventricular pacing. Onpump transcatheter mitral therapy (especially those requiring rapid ventricular pacing) in severely injured LV function may be an optimal procedure, similar to the transcatheter aortic therapy.

\section{Conclusion}

We could successfully perform TM-ViV for a DCM patient with poor LV function, thereby recommending $\mathrm{TM}-\mathrm{ViV}$ as an important treatment option for severely injured LV function.

\section{Disclosure Statement}

Authors have nothing to disclose with regard to commercial support.

\section{Author Contributions}

The conception and design of the work: Koichi Maeda and Yoshiki Sawa.

The acquisition and analysis of data for the work: Koichi Maeda and Isamu Mizote.

Drafting the work: Koichi Maeda and Koichi Toda.

The interpretation of data for the work, revising the work critically for important intellectual content: Toru Kuratani and Yasushi Sakata.

Final approval of the version to be published: all authors.

Accountability for all aspects of the work: all authors.

\section{References}

1) Imamura $T$, Kinugawa $K$, Sakata $Y$, et al: Improved clinical course of autologous skeletal myoblast sheet (TCD-51073) transplantation when compared to a propensity score-matched cardiac resynchronization therapy population. J Artif Organs 2016; 19: 80-86.

2) Chung H, Amaki M, Takashio S, et al: Effect of mitral valve surgery in patients with dilated cardiomyopathy and severe functional mitral regurgitation. Circ J 2018; 82: 131-140.

3) Abe R, Shibata SC, Saito $S$, et al: Factors related to the severity of early postoperative infection after heart transplantation in patients surviving prolonged mechanical support periods: experience at a single university. J Cardiothorac Vasc Anesth 2018; 32: 53-59.

4) Yamashita K, Fukushima S, Shimahara Y, et al: Early outcomes of transcatheter mitral valve replacement 
for degenerated bioprosthesis in Japanese (MITRAL VIV study): a four-case series. Gen Thorac Cardiovasc Surg 2020; 68: 1-8.

5) Blanke P, Naoum C, Dvir D, et al: Predicting LVOT obstruction in transcatheter mitral valve implantation: concept of the Neo-LVOT. JACC Cardiovasc Imaging 2017; 10: 482-485.

6) Maeda K, Kuratani T, Torikai K, et al: On-pump transcatheter aortic valve replacement in patients with poor left ventricular function. J Card Surg 2012; 27: 686-688. 